\title{
Nitabuch's Fibrinoid
}

National Cancer Institute

\section{Source}

National Cancer Institute. Nitabuch's Fibrinoid. NCI Thesaurus. Code C82908.

A protein matrix located between the placenta and the myometrium. 